\title{
ON THE STABILITY OF NEW IMPULSIVE ORDINARY DIFFERENTIAL EQUATIONS
}

\author{
JinRONG WANG — ZENG LIN — YONG ZHOU
}

\begin{abstract}
In this paper, we study new impulsive ordinary differential equations and apply fixed point approach to establish existence and uniqueness theorem and derive an interesting stability result in the sense of generalized $\beta$-Ulam-Hyers-Rassias. At last, two examples are given to demonstrate the applicability of our result.
\end{abstract}

\section{Introduction}

Throughout this paper, $J=[0, T]$ and $C(J, \mathbb{R})$ denotes the space of all continuous functions from $J$ into $\mathbb{R}$. Denote $P C(J, \mathbb{R}):=\{x: J \rightarrow \mathbb{R}: x \in$ $C\left(\left(t_{k}, t_{k+1}\right], \mathbb{R}\right), k=0,1, \ldots, m$ and there exist $x\left(t_{k}^{-}\right)$and $x\left(t_{k}^{+}\right), k=1, \ldots, m$, with $\left.x\left(t_{k}^{-}\right)=x\left(t_{k}\right)\right\}$.

In addition to impulsive differential equations which the impulses are instantaneous, a new class of impulsive differential equations which the impulses are not instantaneous was reported in [9], [17]. It follows [9], [17], a function

2010 Mathematics Subject Classification. 34A37, 34D05, 34D10.

Key words and phrases. Impulsive differential equations, fixed point approach, stability.

The first author acknowledges the support by National Natural Science Foundation of China (11201091) and Outstanding Scientific and Technological Innovation Talent Award of Education Department of Guizhou Province ([2014]240).

The third author acknowledges the support by National Natural Science Foundation of China (11271309), Specialized Research Fund for the Doctoral Program of Higher Education (20114301110001) and Key Projects of Hunan Provincial Natural Science Foundation of China (12JJ2001). 
$x \in V:=P C(J, \mathbb{R}) \bigcap_{i=0}^{m} C^{1}\left(\left(s_{i}, t_{i+1}\right], \mathbb{R}\right)$ is called a classical solution of the following modified impulsive differential equations:

$$
\begin{cases}x^{\prime}(t)=f(t, x(t)), & t \in\left(s_{i}, t_{i+1}\right], i=0,1, \ldots, m, \\ x(t)=g_{i}\left(t, x\left(t_{i}^{+}\right)\right), \quad t \in\left(t_{i}, s_{i}\right], i=1, \ldots, m, \\ x(0)=x_{0} \in \mathbb{R},\end{cases}
$$

if $x$ satisfies $x(0)=x_{0}, x(t)=g_{i}\left(t, x\left(t_{i}^{+}\right)\right), t \in\left(t_{i}, s_{i}\right], i=1, \ldots, m$, and

$$
\begin{array}{ll}
x(t)=x_{0}+\int_{0}^{t} f(s, x(s)) d s, & t \in\left[0, t_{1}\right], \\
x(t)=g_{i}\left(s_{i}, x\left(t_{i}^{+}\right)\right)+\int_{s_{i}}^{t} f(s, x(s)) d s, & t \in\left(s_{i}, t_{i+1}\right], i=1, \ldots, m .
\end{array}
$$

In the present paper, we mainly apply fixed point approach to study a new stability of the following modified impulsive differential equations:

$$
\begin{cases}x^{\prime}(t)=f(t, x(t)), & t \in\left(s_{i}, t_{i+1}\right], i=0,1, \ldots, m, \\ x(t)=g_{i}\left(t, x\left(t_{i}^{+}\right)\right), & t \in\left(t_{i}, s_{i}\right], i=1, \ldots, m,\end{cases}
$$

where $0=t_{0}=s_{0}<t_{1} \leq s_{1} \leq t_{2}<\ldots<t_{m} \leq s_{m}<t_{m+1}=T$ are pre-fixed numbers, $f:[0, T] \times \mathbb{R} \rightarrow \mathbb{R}$ is continuous and $g_{i}:\left[t_{i}, s_{i}\right] \times \mathbb{R} \rightarrow \mathbb{R}$ is continuous for all $i=1, \ldots, m$, which is not instantaneous impulses.

Firstly, we introduce a generalized $\beta$-Ulam-Hyers-Rassias stability concept for the equation (1.2) which is partly motivated by the concepts of stability in [21], [23].

Let $0<\beta \leq 1, \psi \geq 0$ and $\varphi \in P C\left(J, \mathbb{R}_{+}\right)$is nondecreasing. Consider

$$
\begin{cases}\left|y^{\prime}(t)-f(t, y(t))\right| \leq \varphi(t), & t \in\left(s_{i}, t_{i+1}\right], i=0,1, \ldots, m, \\ \left|y(t)-g_{i}\left(t, y\left(t_{i}^{+}\right)\right)\right| \leq \psi, & t \in\left(t_{i}, s_{i}\right], i=1, \ldots, m .\end{cases}
$$

Definition 1.1. The equation (1.2) is generalized $\beta$-Ulam-Hyers-Rassias stable with respect to $(\varphi, \psi)$ if there exists $c_{f, \beta, g_{i}, \varphi}>0$ such that for each solution $y \in V$ of the inequality (1.3) there exists a solution $x \in V$ of the equation (1.2) with

$$
|y(t)-x(t)|^{\beta} \leq c_{f, \beta, g_{i}, \varphi}\left(\psi^{\beta}+\varphi^{\beta}(t)\right), \quad t \in J .
$$

REMARK 1.2. A function $y \in V$ is a solution of the inequality (1.3) if and only if there is $G \in \bigcap_{i=0}^{m} C^{1}\left(\left(s_{i}, t_{i+1}\right], \mathbb{R}\right)$ and $g \in \bigcap_{i=1}^{m} C\left(\left[t_{i}, s_{i}\right], \mathbb{R}\right)$ (which depend on $y$ ) such that:

(a) $|G(t)| \leq \varphi(t), t \in \bigcup_{i=0}^{m}\left(s_{i}, t_{i+1}\right]$ and $|g(t)| \leq \psi, t \in \bigcup_{i=1}^{m}\left(t_{i}, s_{i}\right]$;

(b) $y^{\prime}(t)=f(t, y(t))+G(t), t \in\left(s_{i}, t_{i+1}\right], i=0,1, \ldots, m$;

(c) $y(t)=g_{i}\left(t, y\left(t_{i}^{+}\right)\right)+g(t), t \in\left(t_{i}, s_{i}\right], i=1, \ldots, m$. 
REMARK 1.3. Obviously, if $y \in V$ is a solution of the inequality (1.3) then $y$ is a solution of the following integral inequality:

$$
\left\{\begin{aligned}
\left|y(t)-g_{i}\left(t, y\left(t_{i}^{+}\right)\right)\right| \leq \psi, & t \in\left(t_{i}, s_{i}\right], \quad i=1, \ldots, m \\
\left|y(t)-y(0)-\int_{0}^{t} f(s, y(s)) d s\right| \leq \int_{0}^{t} \varphi(s) d s, \quad t \in\left[0, t_{1}\right] & \\
\left|y(t)-g_{i}\left(s_{i}, y\left(t_{i}^{+}\right)\right)-\int_{s_{i}}^{t} f(s, y(s)) d s\right| \leq \psi+\int_{s_{i}}^{t} \varphi(s) d s & t \in\left(s_{i}, t_{i+1}\right], i=1, \ldots, m .
\end{aligned}\right.
$$

Secondly, we use the same idea and extend to study generalized $\beta$-UlamHyers-Rassias stability of the equation

$$
\begin{cases}x^{\prime}(t)=\lambda x(t)+f(t, x(t)), & t \in\left(s_{i}, t_{i+1}\right], i=0,1, \ldots, m, \lambda>0 \\ x(t)=g_{i}(t, x(t)), & t \in\left(t_{i}, s_{i}\right], i=1, \ldots, m .\end{cases}
$$

Definition 1.4. The equation (1.5) is generalized $\beta$-Ulam-Hyers-Rassias stable with respect to $(\varphi, \psi)$ if there exists $c_{f, \beta, g_{i}, \varphi}>0$ such that for each solution $y \in V$ of the inequality

$$
\begin{cases}\left|y^{\prime}(t)-\lambda y(t)-f(t, y(t))\right| \leq \varphi(t), & t \in\left(s_{i}, t_{i+1}\right], i=0,1, \ldots, m \\ \left|y(t)-g_{i}(t, y(t))\right| \leq \psi, & t \in\left(t_{i}, s_{i}\right], i=1, \ldots, m\end{cases}
$$

there exists a solution $x \in V$ of the equation (1.5) with

$$
|y(t)-x(t)|^{\beta} \leq c_{f, \beta, g_{i}, \varphi}\left(\psi^{\beta}+\varphi^{\beta}(t)\right), \quad t \in J .
$$

Just like Remark 1.3, if $y \in V$ is a solution of the inequality (1.6) then $y$ is a solution of the following integral inequality

$$
\left\{\begin{array}{l}
\left|y(t)-g_{i}(t, y(t))\right| \leq \psi, \quad t \in\left(t_{i}, s_{i}\right], i=1, \ldots, m \\
\left|y(t)-e^{\lambda t} y(0)-\int_{0}^{t} e^{\lambda(t-s)} f(s, y(s)) d s\right| \leq \int_{0}^{t} e^{\lambda(t-s)} \varphi(s) d s \\
\left|y(t)-e^{\lambda\left(t-s_{i}\right)} g_{i}\left(s_{i}, y\left(s_{i}\right)\right)-\int_{s_{i}}^{t} e^{\lambda(t-s)} f(s, y(s)) d s\right| \\
\quad \leq e^{\lambda\left(t-s_{i}\right)} \psi+\int_{s_{i}}^{t} e^{\lambda(t-s)} \varphi(s) d s, \quad t \in\left(s_{i}, t_{i+1}\right], i=1, \ldots, m
\end{array}\right.
$$

For more recent results on Ulam's type stability, the readers can refer to the monographs of [5], [10]-[13], [19], and other works [1], [3], [4], [6], [8], [15], [16], [18], [20], [22], [24] by using fixed point approach and classical analysis methods.

The rest of this paper is organized as follows. In Section 2, Banach fixedpoint theorem for generalized complete metric spaces is used to derive existence and uniqueness of stable solution for the equation (1.2). In Section 3, extension 
type theorem for the equation (1.5) is given. At last, two examples are given to demonstrate the applicability of our result.

\section{Main results}

For a nonempty set $X$, a function $d: X \times X \rightarrow[0, \infty]$ is called a generalized metric on $X$ if and only if $d$ satisfies:

(a) $d(x, y)=0$ if and only if $x=y$;

(b) $d(x, y)=d(y, x)$ for all $x, y \in X$;

(c) $d(x, z) \leq d(x, y)+d(y, z)$ for all $x, y, z \in X$.

Definition 2.1 (see Jung et al. [14] or Balachandran [2]). Suppose $E$ is a vector space over $\mathbb{K}$. A function $\|\cdot\|_{\beta}: E \rightarrow[0, \infty)(0<\beta \leq 1)$ is called a $\beta$-norm if and only if it satisfies:

(a) $\|x\|_{\beta}=0$ if and only if $x=0$;

(b) $\|\lambda x\|_{\beta}=|\lambda|^{\beta}\|x\|_{\beta}$ for all $\lambda \in \mathbb{K}$ and all $x \in E$;

(c) $\|x+y\|_{\beta} \leq\|x\|_{\beta}+\|y\|_{\beta}$.

Theorem 2.2 (see [7]). Let $(X, d)$ be a generalized complete metric space. Assume that $\Lambda: X \rightarrow X$ is a strictly contractive operator with the Lipschitz constant $L<1$. If there exists a nonnegative integer $k$ such that $d\left(\Lambda^{k+1} x, \Lambda^{k} x\right)<$ $+\infty$ for some $x \in X$, then the followings are true:

(a) The sequence $\left\{\Lambda^{n} x\right\}$ converges to a fixed point $x^{*}$ of $\Lambda$;

(b) $x^{*}$ is the unique fixed point of $\Lambda$ in $X^{*}=\left\{y \in X \mid d\left(\Lambda^{k} x, y\right)<\infty\right\}$;

(c) If $y \in X^{*}$, then

$$
d\left(y, x^{*}\right) \leq \frac{1}{1-L} d(\Lambda y, y) .
$$

Next, we introduce the following space of piecewise continuous functions

$$
X=\{g: J \rightarrow \mathbb{R} \mid g \in P C(J, \mathbb{R})\},
$$

endowed with the generalized metric on $X$ defined by

$$
\begin{aligned}
& d(g, h)=\inf \left\{C_{1}+C_{2}\right. \in[0,+\infty] \mid \\
&\left.|g(t)-h(t)|^{\beta} \leq\left(C_{1}+C_{2}\right)\left(\varphi^{\beta}(t)+\psi^{\beta}\right) \text { for all } t \in J\right\},
\end{aligned}
$$

where $C_{1} \in\left\{C \in[0,+\infty]|| g(t)-\left.h(t)\right|^{\beta} \leq C \varphi^{\beta}(t)\right.$ for all $t \in\left(s_{i}, t_{i+1}\right], i=$ $0,1, \ldots, m\}$, and $C_{2} \in\left\{C \in[0,+\infty]|| g(t)-\left.h(t)\right|^{\beta} \leq C \psi^{\beta}\right.$ for all $t \in\left(t_{i}, s_{i}\right]$, $i=1, \ldots, m\}$. It is easy to verify that $(X, d)$ is a complete generalized metric space.

Now, we are ready to study the stability result of the equation (1.2).

TheOrem 2.3. Assume that the following conditions:

$\left(\mathrm{H}_{1}\right) f \in C(J \times \mathbb{R}, \mathbb{R})$; 
$\left(\mathrm{H}_{2}\right)$ There exists a positive constant $L_{f}$ such that

$$
\left|f\left(t, u_{1}\right)-f\left(t, u_{2}\right)\right| \leq L_{f}\left|u_{1}-u_{2}\right|,
$$

for each $t \in J$ and all $u_{1}, u_{2} \in \mathbb{R}$;

$\left(\mathrm{H}_{3}\right) g_{i} \in C\left(\left[t_{i}, s_{i}\right] \times \mathbb{R}, \mathbb{R}\right)$ and there are positive constants $L_{g_{i}}, i=1, \ldots, m$ such that

$$
\left|g_{i}\left(t, u_{1}\right)-g_{i}\left(t, u_{2}\right)\right| \leq L_{g_{i}}\left|u_{1}-u_{2}\right|,
$$

for each $t \in\left[t_{i}, s_{i}\right]$ and all $u_{1}, u_{2} \in \mathbb{R}$;

$\left(\mathrm{H}_{4}\right)$ Let $\varphi \in C\left(J, \mathbb{R}_{+}\right)$be a nondecreasing function. There exists $c_{\varphi}>0$ such that

$$
\int_{0}^{t} \varphi(s) d s \leq c_{\varphi} \varphi(t), \quad \text { for each } t \in J
$$

are satisfied. If there exists a function $y \in V$ satisfing (1.3), then there exists a unique solution $y_{0}: J \rightarrow \mathbb{R}$ such that

$(2.3) y_{0}(t)= \begin{cases}x(0)+\int_{0}^{t} f\left(s, y_{0}(s)\right) d s, & t \in\left[0, t_{1}\right], \\ g_{i}\left(t, y_{0}\left(t_{i}^{+}\right)\right), & t \in\left(t_{i}, s_{i}\right], i=1, \ldots, m, \\ g_{i}\left(s_{i}, y_{0}\left(t_{i}^{+}\right)\right)+\int_{s_{i}}^{t} f\left(s, y_{0}(s)\right) d s, & t \in\left(s_{i}, t_{i+1}\right], i=1, \ldots, m,\end{cases}$

and

$$
\left|y(t)-y_{0}(t)\right|^{\beta} \leq \frac{\left(1+c_{\varphi}^{\beta}\right)\left(\varphi^{\beta}(t)+\psi^{\beta}\right)}{1-\rho}, \quad t \in J
$$

provided that

$$
\rho:=\max \left\{L_{g_{i}}^{\beta}+L_{f}^{\beta} c_{\varphi}^{\beta} \mid i=1, \ldots, m\right\}<1 .
$$

Proof. Define an operator $\Lambda: X \rightarrow X$ by

$$
(\Lambda x)(t)= \begin{cases}x(0)+\int_{0}^{t} f(s, x(s)) d s, & t \in\left[0, t_{1}\right], \\ g_{i}\left(t, x\left(t_{i}^{+}\right)\right), & t \in\left(t_{i}, s_{i}\right], i=1, \ldots, m, \\ g_{i}\left(s_{i}, x\left(t_{i}^{+}\right)\right)+\int_{s_{i}}^{t} f(s, x(s)) d s, & t \in\left(s_{i}, t_{i+1}\right], i=1, \ldots, m .\end{cases}
$$

for all $x \in X$ and $t \in[0, T]$. Clearly, $\Lambda$ is a well defined operator according to $\left(\mathrm{H}_{1}\right)$.

We show that $\Lambda$ is strictly contractive on $X$. Note that the definition of $(X, d)$, for any $g, h \in X$, it is possible to find $C_{1}, C_{2} \in[0, \infty]$ such that

$$
|g(t)-h(t)|^{\beta} \leq \begin{cases}C_{1} \varphi^{\beta}(t), & t \in\left(s_{i}, t_{i+1}\right], i=0,1, \ldots, m, \\ C_{2} \psi^{\beta}, & t \in\left(t_{i}, s_{i}\right], i=1, \ldots, m .\end{cases}
$$


It is easy to see that (2.7) is equivalent to

$$
|g(t)-h(t)| \leq \begin{cases}C_{1}^{1 / \beta} \varphi(t), & t \in\left(s_{i}, t_{i+1}\right], i=0,1, \ldots, m, \\ C_{2}^{1 / \beta} \psi, & t \in\left(t_{i}, s_{i}\right], i=1, \ldots, m .\end{cases}
$$

By the definition of $\Lambda$ in (2.6), $\left(\mathrm{H}_{2}\right),\left(\mathrm{H}_{3}\right)$, and (2.8), we obtain the following three possible cases:

Case 1. $t \in\left[0, t_{1}\right]$, one has

$$
\begin{aligned}
|(\Lambda g)(t)-(\Lambda h)(t)|^{\beta} & =\left|\int_{0}^{t} f(s, g(s)) d s-\int_{0}^{t} f(s, h(s)) d s\right|^{\beta} \\
& \leq L_{f}^{\beta}\left(\int_{0}^{t}|g(s)-h(s)| d s\right)^{\beta} \leq L_{f}^{\beta}\left[C_{1}^{1 / \beta} \int_{0}^{t} \varphi(s) d s\right]^{\beta} \\
& \leq L_{f}^{\beta}\left[C_{1}^{1 / \beta} c_{\varphi} \varphi(t)\right]^{\beta}=L_{f}^{\beta} c_{\varphi}^{\beta} C_{1} \varphi^{\beta}(t) .
\end{aligned}
$$

Case 2. For $t \in\left(t_{i}, s_{i}\right]$,

$$
|(\Lambda g)(t)-(\Lambda h)(t)|^{\beta}=\left|g_{i}\left(t, g\left(t_{i}^{+}\right)\right)-g_{i}\left(t, h\left(t_{i}^{+}\right)\right)\right|^{\beta} \leq L_{g_{i}}^{\beta} C_{2} \psi^{\beta} .
$$

Case 3. For $t \in\left(s_{i}, t_{i+1}\right]$, one can compute

$$
\begin{aligned}
\mid(\Lambda g)( & (t)-\left.(\Lambda h)(t)\right|^{\beta} \\
= & \left|g_{i}\left(s_{i}, g\left(t_{i}^{+}\right)\right)+\int_{s_{i}}^{t} f(s, g(s)) d s-g_{i}\left(s_{i}, h\left(t_{i}^{+}\right)\right)-\int_{s_{i}}^{t} f(s, h(s)) d s\right|^{\beta} \\
\leq & \left|g_{i}\left(s_{i}, g\left(t_{i}^{+}\right)\right)-g_{i}\left(s_{i}, h\left(t_{i}^{+}\right)\right)\right|^{\beta}+\left|\int_{s_{i}}^{t} f(s, g(s)) d s-\int_{s_{i}}^{t} f(s, h(s)) d s\right|^{\beta} \\
& \leq L_{g_{i}}^{\beta}\left|g\left(t_{i}^{+}\right)-h\left(t_{i}^{+}\right)\right|^{\beta}+L_{f}^{\beta}\left[\int_{s_{i}}^{t}|g(s)-h(s)| d s\right]^{\beta} \\
& \leq L_{g_{i}}^{\beta} C_{2} \psi^{\beta}+L_{f}^{\beta}\left[C_{1}^{1 / \beta} \int_{0}^{t} \varphi(s) d s\right]^{\beta} \leq L_{g_{i}}^{\beta} C_{2} \psi^{\beta}+L_{f}^{\beta} C_{1} c_{\varphi}^{\beta} \varphi^{\beta}(t) \\
& \leq\left(L_{g_{i}}^{\beta}+L_{f}^{\beta} c_{\varphi}^{\beta}\right)\left(C_{1}+C_{2}\right)\left(\varphi^{\beta}(t)+\psi^{\beta}\right) .
\end{aligned}
$$

Thus, we have

$|(\Lambda g)(t)-(\Lambda h)(t)|^{\beta} \leq \max \left\{L_{g_{i}}^{\beta}+L_{f}^{\beta} c_{\varphi}^{\beta} \mid i=1, \ldots, m\right\}\left(C_{1}+C_{2}\right)\left(\varphi^{\beta}(t)+\psi^{\beta}\right)$,

for $t \in J$. In other words,

$$
d(\Lambda g, \Lambda h) \leq \rho\left(C_{1}+C_{2}\right)\left(\varphi^{\beta}(t)+\psi^{\beta}\right) .
$$

Hence, we derive

$$
d(\Lambda g, \Lambda h) \leq \rho d(g, h)
$$

for any $g, h \in X$, and since the condition (2.5), the strictly continuous property is shown. 
Let us take $g_{0} \in X$. From the piecewise continuous property of $g_{0}$ and $\Lambda g_{0}$, it follows that there exists a constant $0<G_{1}<\infty$ such that

$$
\begin{aligned}
\left|\left(\Lambda g_{0}\right)(t)-g_{0}(t)\right|^{\beta} & =\left|x(0)+\int_{0}^{t} f\left(s, g_{0}(s)\right) d s-g_{0}(t)\right|^{\beta} \\
& \leq G_{1} \varphi^{\beta}(t) \leq G_{1}\left(\varphi^{\beta}(t)+\psi^{\beta}\right), \quad t \in\left[0, t_{1}\right] .
\end{aligned}
$$

There exists a constant $0<G_{2}<\infty$ such that

$$
\begin{aligned}
\left|\left(\Lambda g_{0}\right)(t)-g_{0}(t)\right|^{\beta} & =\left|g_{i}\left(t, g_{0}\left(t_{i}^{+}\right)\right)-g_{0}(t)\right|^{\beta} \\
& \leq G_{2} \psi^{\beta} \leq G_{2}\left(\varphi^{\beta}(t)+\psi^{\beta}\right), \quad t \in\left(t_{i}, s_{i}\right], i=1, \ldots, m .
\end{aligned}
$$

There exists a constant $0<G_{3}<\infty$ such that

$$
\begin{aligned}
\left|\left(\Lambda g_{0}\right)(t)-g_{0}(t)\right|^{\beta} & =\left|g_{i}\left(s_{i}, g_{0}\left(t_{i}^{+}\right)\right)+\int_{s_{i}}^{t} f\left(s, g_{0}(s)\right) d s-g_{0}(t)\right|^{\beta} \\
& \leq G_{3}\left(\varphi^{\beta}(t)+\psi^{\beta}\right), \quad t \in\left(s_{i}, t_{i+1}\right], i=1, \ldots, m,
\end{aligned}
$$

since $f, g_{i}$ and $g_{0}$ are bounded on $J$ and $\varphi^{\beta}(\cdot)+\psi^{\beta}>0$. Thus, (2.2) implies that

$$
d\left(\Lambda g_{0}, g_{0}\right)<\infty
$$

By using Banach fixed point theorem, there exists a continuous function $y_{0}: J \rightarrow \mathbb{R}$ such that $\Lambda^{n} g_{0} \rightarrow y_{0}$ in $(X, d)$ as $n \rightarrow \infty$ and $\Lambda y_{0}=y_{0}$, that is, $y_{0}$ satisfies equation (2.3) for every $t \in J$.

Next, we check that $\left\{g \in X \mid d\left(g_{0}, g\right)<\infty\right\}=X$. For any $g \in X$, since $g$ and $g_{0}$ are bounded on $J$ and $\min _{t \in J}\left(\varphi^{\beta}(t)+\psi^{\beta}\right)>0$, there exists a constant $0<C_{g}<\infty$ such that $\left|g_{0}(t)-g(t)\right|^{\beta} \leq C_{g}\left(\varphi^{\beta}(t)+\psi^{\beta}\right)$, for any $t \in J$. Hence, we have $d\left(g_{0}, g\right)<\infty$ for all $g \in X$, that is, $\left\{g \in X \mid d\left(g_{0}, g\right)<\infty\right\}=X$. Hence, we conclude that $y_{0}$ is the unique continuous function with the property (2.3). On the other hand, from (1.4) and $\left(\mathrm{H}_{4}\right)$ it follows that

$$
d(y, \Lambda y) \leq 1+c_{\varphi}^{\beta} .
$$

Thus, we derive

$$
d\left(y, y_{0}\right) \leq \frac{d(\Lambda y, y)}{1-\rho} \leq \frac{1+c_{\varphi}^{\beta}}{1-\rho},
$$

which means that (2.4) is true for $t \in J$. The proof is done.

\section{Extension}

Now we adopt the same idea in the above section and extend to study generalized $\beta$-Ulam-Hyers-Rassias stability of the equation (1.5). 
Consider the following impulsive ordinary differential equations with constant coefficients

$$
\begin{cases}x^{\prime}(t)=\lambda x(t)+f(t, x(t)), & t \in\left(s_{i}, t_{i+1}\right], i=0,1, \ldots, m, \lambda>0, \\ x(t)=g_{i}(t, x(t)), & t \in\left(t_{i}, s_{i}\right], i=1, \ldots, m, \\ x(0)=x_{0} \in \mathbb{R} . & \end{cases}
$$

A function $x \in V$ is called a classical solution of (3.1) if $x$ satisfies $x(0)=x_{0}$, $x(t)=g_{i}(t, x(t)), t \in\left(t_{i}, s_{i}\right], i=1, \ldots, m$, and

$$
x(t)=e^{\lambda t} x_{0}+\int_{0}^{t} e^{\lambda(t-s)} f(s, x(s)) d s, \quad t \in\left[0, t_{1}\right],
$$

$x(t)=e^{\lambda\left(t-s_{i}\right)} g_{i}\left(s_{i}, x\left(s_{i}\right)\right)+\int_{s_{i}}^{t} e^{\lambda(t-s)} f(s, x(s)) d s, \quad t \in\left(s_{i}, t_{i+1}\right], i=1, \ldots, m$.

Theorem 3.1. Assume that $\left(\mathrm{H}_{1}\right)-\left(\mathrm{H}_{4}\right)$ are satisfied and a function $y \in V$ satisfies (1.6). Then there exists a unique solution $y_{0}: J \rightarrow \mathbb{R}$ such that

$(3.2) y_{0}(t)=\left\{\begin{array}{lr}e^{\lambda t} x(0)+\int_{0}^{t} e^{\lambda(t-s)} f\left(s, y_{0}(s)\right) d s, & t \in\left[0, t_{1}\right], \\ g_{i}\left(t, y_{0}(t)\right), & t \in\left(t_{i}, s_{i}\right], i=1, \ldots, m, \\ e^{\lambda\left(t-s_{i}\right)} g_{i}\left(s_{i}, y_{0}\left(s_{i}\right)\right)+\int_{s_{i}}^{t} e^{\lambda(t-s)} f\left(s, y_{0}(s)\right) d s, & t \in\left(s_{i}, t_{i+1}\right], i=1, \ldots, m,\end{array}\right.$

and

$$
\left|y(t)-y_{0}(t)\right|^{\beta} \leq \frac{e^{\beta \lambda T}\left(1+c_{\varphi}^{\beta}\right)\left(\varphi^{\beta}(t)+\psi^{\beta}\right)}{1-\rho}, \quad t \in J
$$

provided that

$$
\rho_{\lambda}:=e^{\beta \lambda T} \max \left\{L_{g_{i}}^{\beta}+L_{f}^{\beta} c_{\varphi}^{\beta} \mid i=1, \ldots, m\right\}<1 .
$$

Proof. Just like the proof in Theorem 2.3, we define an operator $\Lambda_{\lambda}: X \rightarrow$ $X$ by

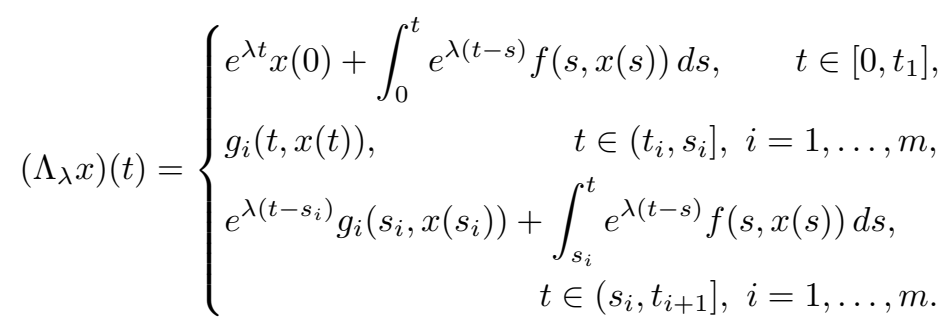

for all $x \in X$ and $t \in[0, T]$.

Clearly, $\Lambda_{\lambda}$ is a well defined operator according to $\left(\mathrm{H}_{1}\right)$. We only need to verify that $\Lambda_{\lambda}$ is strictly contractive on $X$. In fact, it follows from the definition of $\Lambda_{\lambda}$ in (3.5), $\left(\mathrm{H}_{2}\right),\left(\mathrm{H}_{3}\right)$, and (2.8), we obtain that 
Case 1. For $t \in\left[0, t_{1}\right]$,

$$
\left|\left(\Lambda_{\lambda} g\right)(t)-\left(\Lambda_{\lambda} h\right)(t)\right|^{\beta} \leq e^{\beta \lambda T} L_{f}^{\beta} c_{\varphi}^{\beta} C_{1} \varphi^{\beta}(t) .
$$

Case 2. For $t \in\left(t_{i}, s_{i}\right]$,

$$
\left|\left(\Lambda_{\lambda} g\right)(t)-\left(\Lambda_{\lambda} h\right)(t)\right|^{\beta}=\left|g_{i}(t, g(t))-g_{i}(t, h(t))\right|^{\beta} \leq L_{g_{i}}^{\beta} C_{2} \psi^{\beta} .
$$

Case 3. For $t \in\left(s_{i}, t_{i+1}\right]$,

$$
\begin{aligned}
\mid\left(\Lambda_{\lambda} g\right)( & (t)-\left.\left(\Lambda_{\lambda} h\right)(t)\right|^{\beta} \\
\leq & \leq e^{\beta \lambda T} L_{g_{i}}^{\beta}\left|g\left(s_{i}\right)-h\left(s_{i}\right)\right|^{\beta}+e^{\beta \lambda T} L_{f}^{\beta}\left[\int_{s_{i}}^{t}|g(s)-h(s)| d s\right]^{\beta} \\
\leq & e^{\beta \lambda T} L_{g_{i}}^{\beta} C_{2} \psi^{\beta}+e^{\beta \lambda T} L_{f}^{\beta}\left[C_{1}^{1 / \beta} \int_{0}^{t} \varphi(s) d s\right]^{\beta} \\
\leq & \leq e^{\beta \lambda T} L_{g_{i}}^{\beta} C_{2} \psi^{\beta}+e^{\beta \lambda T} L_{f}^{\beta} C_{1} c_{\varphi}^{\beta} \varphi^{\beta}(t) \\
& \leq e^{\beta \lambda T}\left(L_{g_{i}}^{\beta}+L_{f}^{\beta} c_{\varphi}^{\beta}\right)\left(C_{1}+C_{2}\right)\left(\varphi^{\beta}(t)+\psi^{\beta}\right) .
\end{aligned}
$$

From above, we have $d\left(\Lambda_{\lambda} g, \Lambda_{\lambda} h\right) \leq \rho_{\lambda} d(g, h)$, for any $g, h \in X$, and since the condition (3.4), the strictly continuous property is shown.

Let us take $g_{0} \in X$. Proceeding the same procedure in Theorem 2.3, $d\left(\Lambda_{\lambda} g_{0}, g_{0}\right)<\infty$. By using Banach fixed point theorem, there exists a continuous function $y_{0}: J \rightarrow \mathbb{R}$ such that $\Lambda_{\lambda}^{n} g_{0} \rightarrow y_{0}$ in $(X, d)$ as $n \rightarrow \infty$ and $\Lambda_{\lambda} y_{0}=y_{0}$, that is, $y_{0}$ satisfies equation (3.2) for every $t \in J$, which is the unique continuous function.

On the other hand, from $(1.7)$ and $\left(\mathrm{H}_{4}\right)$ it follows that

$$
d\left(y, \Lambda_{\lambda} y\right) \leq e^{\beta \lambda T}\left(1+c_{\varphi}^{\beta}\right) .
$$

Finally,

$$
d\left(y, y_{0}\right) \leq \frac{e^{\beta \lambda T}\left(1+c_{\varphi}^{\beta}\right)}{1-\rho_{\lambda}},
$$

which means that (3.3) is true for $t \in J$. The proof is done.

\section{Example}

In this section, we present two examples, which indicate how our theorems can be applied to concrete problems.

EXAmple 4.1. Consider

$$
\begin{cases}x^{\prime}(t)=\frac{|x(t)|}{8+e^{t}}, & t \in(0,1], \\ x(t)=\frac{\left|x\left(1^{+}\right)\right|}{\left(3+e^{t-1}\right)\left(1+\left|x\left(1^{+}\right)\right|\right)}, & t \in(1,2],\end{cases}
$$


and

$$
\begin{cases}\left|y^{\prime}(t)-\frac{|y(t)|}{8+e^{t}}\right| \leq e^{t}, & t \in[0,1], \\ \left|y(t)-\frac{\left|y\left(1^{+}\right)\right|}{\left(3+e^{t-1}\right)\left(1+\left|y\left(1^{+}\right)\right|\right)}\right| \leq 1, \quad t \in(1,2] .\end{cases}
$$

We put $\beta=1 / 2, J=[0,2]$ and $0=t_{0}=s_{0}<t_{1}=1<s_{1}=2$. Denote

$$
\begin{array}{rlrl}
f(t, x(t)) & =\frac{|x(t)|}{8+e^{t}} & \text { with } L_{f} & =\frac{1}{9} \text { for } t \in[0,1], \\
g_{1}(t, x(t)) & =\frac{\left|x\left(1^{+}\right)\right|}{\left(3+e^{t-1}\right)\left(1+\left|x\left(1^{+}\right)\right|\right)} & \text {with } L_{g_{1}}=\frac{1}{4} \text { for } t \in(1,2] .
\end{array}
$$

We put $\varphi(t)=e^{t}$ and $\psi=1$. Then we choose $c_{\varphi}=1$ satisfying the condition $\int_{0}^{t} e^{s} d s \leq e^{t}$. Moreover, $L_{g_{1}}^{\beta}+L_{f}^{\beta} c_{\varphi}=5 / 6<1$.

Now all the assumptions of Theorem 2.3 are satisfied. Thus, (4.1) has a unique solution $y_{0}:[0,2] \rightarrow \mathbb{R}$ such that $\left|y(t)-y_{0}(t)\right|^{1 / 2} \leq 12\left(e^{t / 2}+1\right)$, for all $t \in[0,2]$.

Example 4.2. Consider

$$
\begin{cases}x^{\prime}(t)=x(t)+\frac{|x(t)|}{35+e^{t}}, & t \in(0,1], \\ x(t)=\frac{|x(t)|}{\left(24+e^{t-1}\right)(1+|x(t)|)}, & t \in(1,2],\end{cases}
$$

and

$$
\begin{cases}\left|y^{\prime}(t)-y(t)-\frac{|y(t)|}{35+e^{t}}\right| \leq e^{t}, & t \in[0,1], \\ \left|y(t)-\frac{|y(t)|}{\left(24+e^{t-1}\right)(1+|y(t)|)}\right| \leq 1, & t \in(1,2] .\end{cases}
$$

Let $\lambda=1, \beta=1 / 2, T=2, J=[0,2]$ and $0=t_{0}=s_{0}<t_{1}=1<s_{1}=2$. Denote

$$
\begin{array}{rlrl}
f(t, x(t)) & =\frac{|x(t)|}{35+e^{t}} & \text { with } L_{f} & =\frac{1}{36} \text { for } t \in[0,1], \\
g_{1}(t, x(t)) & =\frac{|x(t)|}{\left(24+e^{t-1}\right)(1+|x(t)|)} & \text { with } L_{g_{1}}=\frac{1}{25} \text { for } t \in(1,2] .
\end{array}
$$

We put $\varphi(t)=e^{t}$ and $\psi=1$. Set $c_{\varphi}=1$, we have $\int_{0}^{t} e^{s} d s \leq e^{t}$. Obviously,

$$
e^{\beta \lambda T}\left(L_{g_{1}}^{\beta}+L_{f}^{\beta} c_{\varphi}\right)=\frac{11}{30} \times e \approx 0.9968<1 .
$$

By Theorem 3.1, there exists a unique solution $y_{0}:[0,2] \rightarrow \mathbb{R}$ such that

$$
y_{0}(t)=\left\{\begin{array}{cc}
e^{t} x(0)+\int_{0}^{t} e^{t-s} \frac{\left|y_{0}(s)\right|}{35+e^{s}} d s, & t \in[0,1], \\
\frac{\left|y_{0}(t)\right|}{\left(24+e^{t-1}\right)\left(1+\left|y_{0}(t)\right|\right)}, & t \in(1,2],
\end{array}\right.
$$


and

$$
\left|y(t)-y_{0}(t)\right|^{1 / 2} \leq \frac{60 e}{30-11 e}\left(e^{t / 2}+1\right) \quad \text { for all } t \in[0,2] .
$$

Acknowledgements. The authors thank the referees for their careful reading of the manuscript and insightful comments, which helped to improve the quality of the paper. We would also like to acknowledge the valuable comments and suggestions from the editors, which vastly contributed to improve the presentation of the paper.

\section{REFERENCES}

[1] Sz. András and J.J. Kolumbán, On the Ulam-Hyers stability of first order differential systems with nonlocal initial conditions, Nonlinear Anal. 82 (2013), 1-11.

[2] V.K. Balachandran, Topological Algebras, Narosa Publishing House, New Delhi, 1999.

[3] J. BRzdék And S.-M. Jung, A note on stability of an operator linear equation of the second order, Abstr. Appl. Anal. 2011 (2011), Article ID 602713, 15 pages.

[4] M. Burger, N. Ozawa And A. Thom, On Ulam stability, Israel J. Math. 193 (2013), $109-129$.

[5] L. CĂdariu, Stabilitatea Ulam-Hyers-Bourgin Pentru Ecuatii Functionale, Ed. Univ. Vest Timişoara, Timişara, 2007.

[6] D.S. Cimpean and D. Popa, Hyers-Ulam stability of Euler's equation, Appl. Math. Lett. 24 (2011), 1539-1543.

[7] J.B. Diaz And B. Margolis, A fixed point theorem of the alternative, for contractions on a generalized complete metric space, Bull. Amer. Math. Soc. 74 (1968), 305-309.

[8] B. Hegyi and S.-M. Jung, On the stability of Laplace's equation, Appl. Math. Lett. 26 (2013), 549-552.

[9] E. Hernández and D. O'Regan, On a new class of abstract impulsive differential equations, Proc. Amer. Math. Soc. 141 (2013), 1641-1649.

[10] D.H. Hyers, On the stability of the linear functional equation, Proc. Nat. Acad. Sci. 27 (1941), 222-224.

[11] D.H. Hyers, G. Isac And Th. M. Rassias, Stability of Functional Equations in Several Variables, Birkhäuser, 1998.

[12] S.-M. Jung, Hyers-Ulam-Rassias Stability of Functional Equations in Mathematical Analysis, Hadronic Press, Palm Harbor, 2001.

[13] _ Hyers-Ulam-Rassias Stability of Functional Equations in Nonlinear Analysis, Springer, New York, 2011.

[14] S.-M. Jung, T.S. KIm And K.S. LeE, A fixed point approach to the stability of quadratic functional equation, Bull. Korean Math. Soc. 43 (2006), 531-541.

[15] S.-M. Jung And Th.M. Rassias, Generalized Hyers-Ulam stability of Riccati differential equation, Math. Inequal. Appl. 11 (2008), 777-782.

[16] N. Lungu And D. Popa, Hyers-Ulam stability of a first order partial differential equation, J. Math. Anal. Appl. 385 (2012), 86-91.

[17] M. Pierri, D. O'Regan And V. Rolnik, Existence of solutions for semi-linear abstract differential equations with not instantaneous impulses, Appl. Math. Comput. 219 (2013), 6743-6749.

[18] D. Popa And I. Raşa, On the Hyers-Ulam stability of the linear differential equation, J. Math. Anal. Appl. 381 (2011), 530-537. 
[19] Th.M. Rassias, On the stability of linear mappings in Banach spaces, Proc. Amer. Math. Soc. 72 (1978), 297-300.

[20] H. Rezaei, S.M. Jung and Th.M. Rassias, Laplace transform and Hyers-Ulam stability of linear differential equations, J. Math. Anal. Appl. 403 (2013), 244-251.

[21] I.A. Rus, Ulam stability of ordinary differential equations, Studia Univ. "Babeş Bolyai" Mathematica 54 (2009), 125-133.

[22] Ulam stabilities of ordinary differential equations in a Banach space, Carpathian J. Math. 26 (2010), 103-107.

[23] J. WANG, M. FEČKAN AND Y. Zhou, Ulam's type stability of impulsive ordinary differential equations, J. Math. Anal. Appl. 395 (2012), 258-264.

[24] T.Z. Xu, On the stability of multi-Jensen mappings in $\beta$-normed spaces, Appl. Math. Lett. 25 (2012), 1866-1870.

Manuscript received October 5, 2013

JiNRONG WANG AND ZENG LIN

School of Mathematics and Computer Science

Guizhou Normal College

Guiyang, Guizhou 550018, P.R. CHINA

E-mail address: wangjinrong@gznc.edu.cn, linzeng822@126.com

YONG ZHOU

Department of Mathematics

Xiangtan University

Xiangtan, Hunan 411105, P.R. CHINA

E-mail address: yzhou@xtu.edu.cn 